• 保护论坛・

\title{
《生物多样性公约》企业与生物多样性全球 平台的发展情况及对中国的政策建议
}

\author{
赵 $\quad$ 阳 ${ }^{1}$ 温源远 ${ }^{1,2 *}$ \\ 1 (生态环境部环境保护对外合作中心, 北京 100035) \\ 2 (北京师范大学环境学院, 北京 100875 )
}

\begin{abstract}
摘要: 《生物多样性公约》(以下简称《公约》) 是国际社会协力保护生物多样性最主要的公约, 也是中国参与和引 领全球生态文明建设的重要平台。《公约》一直不断推动企业参与生物多样性保护的相关工作和国际体系建设。 该国际体系主要包括两大支柱: 一是“企业与生物多样性全球伙伴关系”国际机制(Global Partnership on Business and Biodiversity, 以下简称GPBB机制); 二是“企业与生物多样性全球伙伴关系”资源平台(Global Platform for Business and Biodiversity, 以下简称GPBB平台)。前者是工作组织和资金机制，后者是前者的技术支持和保障机制， 二者相辅相成, 协同增效。总体来看, 自1996年《公约》第三次缔约方大会(COP-3)以来, GPBB平台的相关概念和 机制不断发展完善, 至今已形成了一个GPBB平台在线数据库, 可为我国推进相关工作提供技术支撑。通过梳理总 结GPBB平台及其相关机制等的发展情况, 得出: (1)企业参与生物多样性保护的意愿及对GPBB平台的需求日益增 强; (2)《公约》对企业参与的要求更加明确; (3)《公约》对如何促进企业参与生物多样性的路线图更加清晰; (4) GPBB机制已基本建立; (5) GPBB平台已基本完善。展望未来, GPBB平台发展将有望继续保持以下三大趋势: (1)平 台建设力度将不断加强; (2)平台的影响将不断提升; (3)平台发展将向重点部门倾斜。目前, GPBB平台可提供相关 最新研究成果、方法学、工具、自愿性标准和指引、简报、最佳实践案例等免费信息, 以及一系列项目实施过程 中的企业参与机会, 以支持企业在决策和运营中纳入生物多样性, 并鼓励支持各国政府、企业、非政府组织和学 术科研机构等多利益相关方共同参与、贡献与受益。中国自2015年正式加入GPBB机制后，对各成员国的企业参 与倡议开展了较为深入的研究, 为成立发起“中国企业与生物多样性伙伴关系”(CBBP)联盟倡议做出有益探索。但 总体而言, 对GPBB平台的研究借鉴以及参与建设还有很多工作可做。我们建议: (1)在制度贡献方面, 应加强政策 研究, 研提COP-15企业参与新决议草案; (2)在机制建设方面, 积极参与和逐渐引领GPBB机制建设, 协力支撑一 带一路等中国倡议; (3)在人员保障方面, 加强人才队伍建设, 提升引领能力; (4)在GPBB平台方面, 加强研究借鉴 和技术参与, 为GPBB平台建设提供技术支持。
\end{abstract}

关键词：生物多样性保护; 国际经验; 全球伙伴关系; 生物多样性公约; 企业参与

\section{Development of Convention on Biological Diversity's Global Platform for Business \& Biodiversity: Policy suggestion for China}

\author{
Yang Zhao ${ }^{1}$, Yuanyuan Wen ${ }^{1,2 *}$ \\ 1 Foreign Economic Corporation Office, Ministry of Ecology and Environment of People's Republic of China, Beijing \\ 100035 \\ 2 School of Environment, Beijing Normal University, Beijing 100875
}

\begin{abstract}
The Convention on Biological Diversity (CBD) is the most important international convention committed to the protection of biodiversity. It is also the most significant platform for China to participate and lead in the construction of a global ecological civilization. As an important entity for enterprises engaging in biodiversity conservation, the CBD has been strengthening efforts to build a consistent international system over the last two decades. The international system consists of two main pillars: (1)
\end{abstract}


Global Partnership on Business and Biodiversity (GPBB mechanism), and (2) Global Platform for Business \& Biodiversity (GPBB platform). The GPBB mechanism provides the organization and financial arrangement, while the GPBB platform mainly provides technical and intellectual support. These two pillars complement and provide synergies to one other. By looking into the formation background, development process, current situation and future trends of the GPBB platform, this paper systematically summarizes various resources that have been intentionally made available by the GPBB platform gradually over a long period of time. It also puts forward relevant policy recommendations for China in how to better participate in, contribute to and benefit from the CBD. Since COP-3 in 1996, related concepts, methodologies, and mechanisms have been continuously built up and made publicly available through an online database of the current GPBB platform through the official website of the Convention. These resources can be used by governments and enterprises of all countries, and China should increase its application of the resources available. Based on our research, we came to the following conclusions: (1) Willingness of enterprises to participate in biodiversity conservation and the demand for the GPBB platforms is growing; (2) CBD's requirements for enterprise participation are explicit; (3) Roadmap on how to promote business engagement in biodiversity protection is straightforward; (4) GPBB mechanism allowing national governments to become members has already been established; and (5) GPBB platform as resource bank has been greatly substantiated. Looking forward to the future, the development of GPBB platforms is expected to continue maintaining the following three momentums: (1) GPBB platform will be continuously improved; (2) The impact will be further strengthened; and (3) More significant sectors will be highlighted. The GPBB platform has provided opportunities for enterprises to implement a range of CBD projects and promoted up-to-date information dissemination on latest research findings, methodology, technical tools, voluntary standards, guidelines, briefings and business best practices. It has also supported inputs and contributions from GPBB members of national governments, corporations, NGOs, academic researchers and other institutional stakeholders. China formally joined the GPBB in 2015 and carried out an in-depth study on GPBB and membership countries. A national initiative of China Business \& Biodiversity Partnership (CBBP) is in progress, however in general, there is still much work to do. The paper makes the following suggestions: (1) China should introduce new draft resolutions to further strengthen the international system in COP-15 based on improved policy research; (2) China should be active in and gradually lead the GPBB development; (3) Capacity building in human resources is needed to make the CBBP stand out in the GPBB; and (4) Draw on the GPBB platform to better benefit from and contribute to it.

Key words: biodiversity conservation; international experience; global partnership; Convention on Biological Diversity (CBD); business participation

《生物多样性公约》(以下简称《公约》) 是国 际社会协力保护生物多样性的最重要公约(庄国泰 和沈海滨, 2013)。新时期下, 大力创新推动《公约》 履约相关工作, 是深入贯彻落实十九大精神, 推进 国内生态环境保护和生态文明建设, 引领全球生态 文明建设的重要方面。企业是开发和利用生物多样 性的主体, 也是实现生物多样性可持续发展必不可 少的推动力量(张风春等, 2014)。为推动企业参与, 《公约》根据历届缔约方大会决议要求, 通过完善 制度、体制、机制和资源网络, 逐步建立了促进企 业参与生物多样性保护的国际体系。该体系由两大 支柱构成: 一是作为国际机制的“企业与生物多样 性全球伙伴关系”(Global Partnership on Business and Biodiversity, 简称 GPBB机制); 二是作为公共 在线资源网络的“企业与生物多样性全球平台”(Gl- obal Platform for Business and Biodiversity, 简称 GPBB平台)。前者是工作组织和资金机制, 后者是 前者的技术支持和保障机制，二者相辅相成，协同 增效。

关于GPBB机制的发展情况及国际经验, 赵阳 等(2018b)已有较为具体的介绍。简言之, 为加强生 物多样性保护, 国际社会逐渐形成共识, 需要全社 会共同努力以保护生物多样性，尤其是企业的参 与。《公约》也不断推进企业参与生物多样性保护 工作。1996年召开的公约缔约方大会第三次会议 (COP-3)首次提出了企业参与生物多样性的概念(王 爱华等, 2015); 2000年召开的COP-5将企业参与列 入《公约》议题; 2002年的COP-6将企业参与纳入《公 约》的战略内容; 2006年的COP-8首次将企业参与生 物多样性单独纳入 《公约》决议; 2009年的COP-9 
拟定了首个企业参与行动框架; 2010年的COP-10将 企业参与纳入战略目标, 并要求国家和区域层面制 定相关倡议和努力建设GPBB；2012年COP-11通过 的决议要求利用GPBB为框架促进企业界、政府和 其他利益敒关方之间的对话; 2014年COP-12的决议 要求与GPBB及其相关国家和区域倡议协作以支持 企业界的能力建设; 2016年COP-13发起了《企业与 生物多样性承诺书》倡议。笔者通过分析德国、印 度、加拿大、秘鲁、澳大利亚、南非、日本和韩国 构建企业参与GPBB的实践情况, 发现主要有以下 特点: (1)政府发挥着重要的指引作用和服务功能; (2)各国工作和资金筹措方式多样; (3)成员加入需要 签署《生物多样性保护宣言》; (4)推动企业参与的 GPBB平台已基本建立; (5)大量企业表现出参与生 物多样性伙伴关系倡议的积极意愿。

以下将重点介绍GPBB平台的发展情况及对中 国的政策建议。GPBB平台的建设流程是: (1)各国加 入GPBB后, 向《公约》提交成果和知识产品; (2)这 些成果和知识产品经审核后, 正式纳入GPBB平台 供其他国家或地区借鉴。经多年逐步充实发展, GPBB平台已成为为成员国提供技术和智力支持、 企业示范机会和最佳实践展示窗口的综合性资源 网络, 辐射并惠益全球各地企业。本文首先介绍公 约GPBB平台的相关发展进程和现状, 然后分析中 国相关工作的进展, 最后研提对中国的政策建议, 为充分利用GPBB平台建立健全国内相关工作机制, 办好 2020 年 《公约》缔约方大会第十五次会议 (COP-15)提供参考。

\section{GPBB平台的形成与发展}

\subsection{GPBB平台的发展进程}

2010年COP-10提出建立GPBB平台, 为落实相 关决议要求, 《公约》决定开展全球企业参与多样性 对话论坛, 并于2011年12月在日本举行了“全球企 业界参与生物多样性平台论坛” 第一次会议 (GPBB-1)。2012年10月COP-11召开期间, 该论坛举 行第二次会议(GPBB-2)并改名为 “全球企业界参与 生物多样性伙伴关系论坛”。2013年10月, GPBB-3 在加拿大召开, 中国代表第一次受邀参加。2014年 10月, COP-12会议期间, GPBB-4召开并宣布成立 GPBB执行委员会。2014年《公约》秘书处正式成 立了GPBB。目前已有包括中国在内的17个国家和3
个区域(欧盟、东南亚与中美洲)加入该机制。

GPBB机制和GPBB平台是相伴相生的, 在不 断推进GPBB的同时, 《公约》也对GPBB平台的建 设提出了一系列要求。《公约》促进GPBB机制内 的各成员国政府、国际组织、企业、NGO和学术科 研机构等多利益相关方参与、贡献并受益于该公共 资源网络, 提供国际最新研究成果、方法学、工具、 自愿性标准、简报、案例和数据库, 以及一系列项 目实施过程中的企业参与机会, 支持企业在决策和 运营中纳入生物多样性。例如, 2010年COP-10通过 《X/21企业界的参与》决议, 要求“编辑最佳做法, 促进企业界参与采用这些做法”并“改进各种机制和 工具, 帮助企业界理解、评估和采取管理生物多样 性风险的解决方案”。2012年COP-11通过的《生物 多样性促进消除贫困和发展》和《XI/7企业界与生 物多样性》决议则要求 《公约》秘书处成立为国家 和区域提供分享和对话的全球平台, 促进私营部门 主流化。2014年COP-12《XII/10企业界的参与》决 议则强调要“鼓励工商界分析企业决策和运营对生 物多样性和生态系统功能和服务的影响, 并编制将 生物多样性纳入其运营的行动计划”。

2017年, 经秘书处多年争取, 《公约》批准新 设一个企业事务项目官员的长期职位, 主要负责落 实《公约》两大支柱工作：一是持续发展GPBB的国 家与区域成员; 二是继续实施一系列项目, 促进企 业参与、贡献和受益于 GPBB平台。

\subsection{GPBB平台的总体现状}

通过梳理总结, GPBB平台现状有以下特点:

(1)企业参与生物多样性保护的意愿及对GPBB 平台的需求日益增强。生物多样性维系着人类经济 发展、社会福祉和个人生计, 提供人类所需的食品、 建筑材料、医药和能源等自然资本已成为社会共识, 它已从单一的环境议题提升为可持续发展主题, 这 使得企业更为重视承担相关的社会责任, 维护其社 会声誉。国际社会将生态系统服务指标纳入责任投 资、绿色供应链、可持续消费、战略环评、公共采 购、信息披露、生态城市等相关国际或本国倡议、 标准和指引中, 企业也逐步意识到生物多样性保护 对其获得相关认证认可、特许经营、许可证和配额 等存在的潜在风险与机会, 正加快推动企业核算生 态系统服务价值、评估对生物多样性的影响与依赖, 并披露相关信息。 
(2)对企业的要求更加明确。以前《公约》要求 企业“采取切实行动, 将生物多样性纳入到运营与 决策当中”, 目前则进一步要求: “不论出于规避风 险或寻求商业机会的动机, 还是考虑业务流程或价 值链，生物多样性都是关键要素”。

(3)对如何促进企业参与生物多样性的路线图 更加清晰。即“支持企业学习、应用，帮助企业了解、 评价与核算对生物多样性、生态系统服务的依赖和 影响以及相关风险与机会”。

(4)引导企业参与生物多样性保护的国际机制 (GPBB)已基本建立。GPBB承上启下，弥合了国际 《公约》与微观企业间的鸿沟。特别是很多国家的 加入, 不仅带动了本国企业的参与, 通过试点示范 和推动拉动因素的倍增效应, 还将辐射和影响世界 各地企业的意识、态度和行为, 深度参与和不断加 强生物多样性保护工作。

(5)支持企业参与的GPBB平台已基本完善。企 业参与的实际需求须运用具体知识产品, 包括理论 方法学、技术工具和实践案例等, 同时要遵循法律 法规、部门监管、认证认可、行业指引和市场标准 等不同层面的自愿性标准。《公约》通过其官网数 据库已提供关于上述内容的资源与试点机会。目前 GPBB平台的网络工具已非常系统，仅 “工具/机制” 一个分类, 就已包括核算、缓解、抵偿、报告、指 引、法规、认证和监测与评估等一百多种工具。

\subsection{GPBB平台的发展趋势}

展望未来, GPBB平台的发展将有望继续保持 以下三大趋势:

(1) GPBB平台建设力度将不断加强。 《公约》 将继续推动更多国家加入GPBB机制, 其作用和意 义必将在新缔约方大会的决议中得到巩固和加强。 随着GPBB机制的加强, GPBB平台的建设力度将不 断增强。

(2) GPBB平台的作用将不断增大。 《公约》将 继续推动各成员国在GPBB平台上提交成果，共同 分享, 相互借鉴并加强合作, 提高各国服务企业的 能力水平和作用。其中, 评估企业对生态系统服务 和自然资本的依赖和影响, 披露信息与传播企业最 佳实践, 是促进全球企业参与履行 《公约》和《联 合国可持续发展议程》的重要技术路线。

(3) GPBB平台发展将向重点部门倾斜。根据 《公约》谈判达成的有关共识, GPBB与GPBB平台
将继续对重点企业部门倾斜，主要有三类：生态影 响型(如采掘、水电等)、资源利用型(如生物勘探、 制药、化妆品、农林渔等)，以及议定书特定议题相 关行业(如检验检疫等)。

\section{GPBB平台所提供的主要信息和}

根据《公约》决议要求, 在《公约》官网上开 设了GPBB平台专栏，提供国际最新研究成果、方法 学、工具、自愿性标准、简报、案例和数据等免费 信息，以及一系列项目实施过程中的企业参与机会， 支持企业在决策和运营中纳入生物多样性，鼓励支 持各国政府、企业、非政府组织和学术科研机构等 多利益相关方共同参与、贡献并受益(张风春等, 2015)。

《公约》在GPBB平台专栏首页提出的相关商 业风险包括：资源稀缺、合规监管、责任投资、生 态补偿所带来的配额、成本、公司形象受损甚至产 品遭受抵制等; 机会包括：市场份额增加、迎合责 任消费、利益相关方沟通改善、员工忠诚度提高、 技术和产品创新、更精益或可持续的生产工艺或商 业模式等。专栏建议: “企业采取切实行动，将之纳 入到运营与决策当中”; “提供信息知识、技术工具、 最佳实践案例、研究成果、方法学和自愿性标准以 及可参与的项目、宣传对话等资源和渠道，支持企 业学习、应用，帮助企业了解、评价与核算对生物 多样性、生态系统服务的依赖和影响以及相关风险 与机会”。

目前GPBB平台主要提供以下资源和参与机会: 2.1 开发企业贡献《爱知目标》的最佳实践案例, 并制定企业参与通用导则

针对企业对国际议程缺乏了解而无法参与的 呼声, COP-12《XII/10企业界的参与》决议3(d)要求: “与企业界和生物多样性全球伙伴关系及相关国家 和区域倡议协作，通过查明关键的进度指标和为企 业界制定支持执行《生物多样性战略计划》 (2011-2020年)的指导方针, 推动企业部门对实现爱 知生物多样性目标做出贡献”。目前秘书处正在实 施“制定企业参与实现《爱知目标》的商业框架和 案例项目”, 旨在向工商界解释爱知生物多样性目 标并提供指导建议，支持企业为贡献这些目标、指 标采取措施，对已采取了一项或多项爱知目标相关 行动的公司进行一系列案例研究, 总结经验教训, 
传播最佳实践案例，制定通用技术导则，促进私营 部门对《公约》议程的认知和参与。

\section{2 激励企业核算生物多样性价值, 并推荐方法 学与工具}

2014年《全球环境变化杂志》报告指出, “经核 算, 世界生态系统服务总价值已达到全球GDP的两 倍, 即每年 124.8 万亿美元”。2014年COP-12《XII/10 企业界的参与》决议3(f)要求: “汇编有关生物多样 性和生态系统功能和服务以及这些服务的价值的 信息, 并分析这方面的最佳做法、标准和研究, 促 进评估企业界对实现《公约》各项目标和爱知生物 多样性目标的贡献, 并协助向各相关论坛传播这些 信息”。这将生物多样性、生态系统服务价值评估 与自然资本核算直接联系起来，已获得全球各界， 尤其工商企业界的广泛认同。《公约》推荐由自然 资本联盟(Natural Capital Coalition)、联合国环境规 划署、IUCN和世界可持续发展工商理事会(WBCSD) 共同发起了 “世界自然资本论坛”, 以便于企业获取 更多信息与技术。目前各国倡议多有运用和引进

《自然资本议定书》 (Natural Capital Protocol), 帮助 本国企业评估其对生态系统服务的影响和依赖, 以 及相关风险与机会。生态环境部环境保护对外合作 中心(Foreign Economic Cooperation Office, FECO) 作为“中国企业与生物多样性伙伴关系”(CBBP)的 管理和运营机构, 目前正在翻译和出版该议定书, 推动国内试点行业和企业实施。

\section{3 鼓励企业披露信息, 并发起《企业与生物多样 性承诺书》倡议}

企业运用工具、方法学和案例, 了解、评估与 核算对生物多样性、生态系统服务的影响、依赖和 价值后，针对如何报告相关信息的问题，2014年 COP-12《XII/10企业界的参与》决议强调: “鼓励工 商界分析企业决策和运营对生物多样性和生态系 统功能和服务的影响, 并编制将生物多样性纳入其 运营的行动计划”; “将与生物多样性和生态系统功 能和服务相关的问题纳入其报告框架, 确保了解公 司所采取的行动，包括通过其供应链，同时考虑到 《公约》的各项目标、《生物多样性战略计划》 (2011-2020年)和爱知生物多样性目标”; “企业与生 物多样性全球伙伴关系与相关的国家和区域倡议 合作，以便协助企业界报告其实施《公约》及其议 定书的目标以及战略计划的进展”; 敦请执行秘书
“支持企业界与生物多样性全球伙伴关系及其相关 国家和区域倡议并与其协作，编制关于企业界将生 物多样性纳入主流的进度报告”。公约秘书处则通 过企业承诺书等倡议，号召世界各地的企业报告履 行承诺所采取的行动和进展。

2016年墨西哥召开的COP-14发起了《企业与生 物多样性承诺书》倡议，已有 100 多个来自不同国 家、多个部门的企业和金融机构签署加入。中国生 态环境部环境保护对外合作中心推动伊利集团加 入了该倡议。《承诺书》指出: “......可持续的消费 和生产模式，可为企业带来切实的利益，包括更加 可靠的材料和产品供应、节约成本和自然灾害防 护。这种转变是实现《生物多样性战略计划》以及 执行《2030年可持续发展议程》及其可持续发展目 标的必要条件”。《承诺书》呼吁: “了解、计量并在 可行的情况下估算公司对生物多样性和生态系统 服务的影响和依赖”, “制定生物多样性管理计划, 包括管理供应链的举措”, “定期报告公司对生物多 样性和生态系统服务的影响和依赖”，并“将生物多 样性因素更好地纳入企业决策”。目前秘书处已开 发了问卷和报告格式，供签署企业定期报告履行承 诺的进展情况。

\section{4 发布大宗商品生产的生物多样性影响指标与 指南}

2014年, COP-12《XII/10企业界的参与》决议 3(e)明确提出: “促进与其他论坛在与生物多样性和 企业界参与相关问题上进行指标和可持续生产和 消费方面的合作和协同增效”。针对目前许多国家 栖息地丧失的主要原因是土地用途转做农产品生 产的事实与挑战，《公约》研究了不同国家与地区 不同种类作物对不同生态系统服务的影响和依赖, 纳入了相关主要国际自愿性可持续标准，编制了 《自愿性标准与生物多样性报告》。该报告研究了 不同国家和地区的8种农产品(茶、甘蔗、可可、棉 花、咖啡、谷物、棕榈树和大豆), 并从水质、水体 富营养化、土壤肥力、生物需氧量、温室气体排放、 用水量、自然植被丧失、栖息地质量、减缓气候变 化、土地管理、燃料等生态系统服务方面, 编制了 一套基础性、普适性农产品生产对生物多样性影响 指标和指南, 可作为企业最低生物多样性绩效标准, 帮助企业更好地理解产品生命周期所涉及的生态 价值、风险影响和外部不经济性。例如，该报告指 
出, 气候变化和土地用途(由保护地变更为农业生 产用地) 是发展中国家生物多样性丧失的最大驱动 力。当前, 大部分自愿性标准都已纳入了关于温室 气体排放、土地用途公共治理以及其他可持续发展 指标，所以基于市场的认证认可有利于预防和减缓 生物多样性丧失。例如, 非洲土地肥力处于持续下 降趋势, 而良好的可可、咖啡种植标准要求“树荫下 间作”, 这种农林混作模式无疑有利于保护森林。再 例如: 《更好的棉花倡议》(Better Cotton Initiative, BCI)标准强调水循环利用和高效灌溉，2014年已认 证棉花产量达190万吨，占全球棉花总产量的7\%。 棕㭣油、大豆的需求旺盛造成马来西亚、印尼和南 美洲的自然栖息地改作种植园，而认证有利于遏制 这一趋势。

\section{5 发布《卡塔赫纳生物安全议定书》相关技术信息}

资源网站提供了知识产品、出版物、简报和信 息交换所等免费资源，帮助企业了解《卡塔赫纳生 物安全议定书》所涉及的关于现代生物技术产生的 “改性活生物体”(LMO)跨境转移所造成的损害责 任、补救和赔偿等运营风险，以及包括抽样与检测、 运输和包装、风险评估与管理、向监管部门报告等 具体措施。针对企业发布了《生物安全技术系列工 具》，包括《改性活生物体运输标准》、《审查损害 赔偿责任和补救工具》、《改性活生物体风险评估和 监测指南》和《九个国家风险评估方法摘要和比较 分析》等技术工具与指南。

\section{6 发布《名古屋议定书》相关技术信息}

资源网站提供了知识产品、出版物、简报和信 息交换所等免费资源，帮助企业了解《名古屋议定 书》关于获取遗传资源并公平和公正地分享其利用 所产生惠益所涉及的 “事先知情同意”(Prior Informed Consent, PIC)、“共同商定条件” (Mutually Agreed Terms, MAT)和 “相关传统知识”(Associated Traditional Knowledge, ATK)等概念, 国际公约、各 国立法和政府监管等进展情况, 特许经营、许可证 和配额等市场手段, 企业最佳实践、成功故事的案 例分析等。针对不同部门的企业发布了一系列政策 简报，涵盖农业、植物制剂、化妆品、食品与饮品、 工业生物技术和制药等多个行业，并提供中文等多 个语言版本。所有信息目前被秘书处整合在生物多 样性获取与惠益分享(ABS) 倡议网站中, 分为出版 物、知识中心、指南、案例研究和视听资料等部分,
并提供搜索功能。

\section{7 建立在线数据库并提供信息搜索与分类服务}

资源网站将其数据库建立在《公约》官网上，囊 括了全球范围行之有效支持企业开展生物多样性 和生态系统服务相关的工具/机制(289个)、案例研 究(45个)、信息简报(21个)、组织机构(428个)、会 议活动等免费信息资源，并提供分类与搜索功能。

\subsection{1 工具/机制}

在众多工具中， 《自然资本核算体系》排在搜 索首位，显示出其应用已是国际大势所趋。页面提 供索引功能, 可按照部门(包括农、林、渔、采掘、 能源、基建、化妆品、银行、旅游等十几个行业) 和国家进行搜索，主要包括以下几类工具：(1)减缓 (Mitigation), 例如《缓解层次实施导则》; (2)核算 (Valuation), 例如《企业生态系统服务核算》; (3)信 息披露(Reporting), 例如《生物多样性作为企业报告 资源指引》; (4)技术指南(Guideline), 例如《生物多 样性私营部门指南》; (5)指引(Guidebook), 例如《海 岸自然资本核算指南》; (6)认证认可(Certification \& Accreditation), 例如 《可持续渔业行为准则》和《最 佳水产养殖实践认证》; (7)跨部门综合工具, 例如 《企业与生物多样性》; (8)特定行业工具, 例如《石 油天然气行业水工具》和《水泥行业生物多样性管 理计划指南》。引擎可搜索到与日本相关的内容 9 个, 都与机制(机构)相关，且大多是非政府组织，例如 致力于有机稻米认证的日本生态系统农业协会、日 本生态旅游协会、促进负责任金枪鱼渔业组织、国 家林业管理改进和传播组织、可持续能源政策研究 所、市民环境基金会等。但搜索中国结果为零。

\subsection{2 案例研究}

与工具一样, 案例研究也提供了国家与行业分 类搜索，包括全球各地企业参与生物多样性的成功 故事。例如陶氏化学运用自然资本核算遴选最佳污 水处理工艺(赵阳和王影, 2018), 力拓集团将生物多 样性保护提升到公司战略的系列行动(赵阳等, 2018a), 联合利华在农业标准中纳入生物多样性指 标，卡特彼勒、塔塔钢铁和施乐等公司将生态系统 服务作为实施《联合国可持续发展议程》重要途径 等最佳实践。引擎可搜索到日本相关内容 9 个, 包括 小型树栖动物迁移廊道、塞舌尔群岛珊瑚礁保护、 利用钢铁生产中废渣和林业废弃木片恢复沿海生 态系统、无农药棉花农场等企业项目案例研究报 
告。而搜索中国结果仍为零。

\subsection{3 相关组织}

数据库提供的相关组织数据也提供了国家与 行业的分类搜索，部分与“工具/机制”重合。里面既 有IUCN、WBCSD、WRI、WWF、FAO、GIZ等国 际机构, 也有全球报告倡议组织(GRI)、森林管理委 员会(FSC)、公平贸易(Fair Trade)等标准组织, 也有 一批不知名的地方本土非政府组织。自然资本联盟 排在首页，他们开发的《自然资本核算体系》已取 代《生态系统服务与生物多样性经济学》(TEEB), 具 有国际权威地位。经测试, 可搜索到12个日本组织, 包括几乎与中国同时加入 《公约》 “全球伙伴关系” 的“日本企业倡议”(Japan Business Initiative for Biodiversity, JBIB)。而搜索中国为零。

\subsection{4 《公约》企业简报}

数据库提供了《公约》企业简报。简报每期侧 重不同主题, 如当年的缔约方大会(COP)、《名古屋 议定书》、《联合国可持续发展议程》(SDG) 和气候 变化等; 或特定领域, 如金融、采掘和生态旅游等; 或活跃国家，如加拿大、印度等。

\subsection{5 会议和活动}

数据库提供了秘书处和其他国际机构组织的 相关论坛、研讨会和活动信息, 2018年最重大活动 是11月7-8日在埃及举办的《公约》“企业全球论坛”。

\section{3 中国加入 GPBB机制和利用GPBB平台的 情况}

2015年, 中国正式加入GPBB国际机制。生态环 境部授权环境保护对外合作中心(FECO)负责构建 “中国企业与生物多样性伙伴关系”(CBBP)。FECO 对各成员国的企业倡议, 从政府职能、组织架构、 成员招募、技术服务、资金平衡和主要挑战等方面 开展了深入研究, 为成立发起CBBP倡议, 推动企 业参与做出了有益探索和持续努力。包括借鉴《公 约》GPBB平台上的资源, 开展了国内TEEB研究工 作, 在中医药行业推广价值评估的试点项目; 为国 家标准委和商务部五矿化工商会在《社会责任标准 GB36000》与《海外矿业投资社会责任指引》中纳 入生物多样性保护的原则提供技术支持；2015年 FECO支持浙江省仙居国家公园成功获得法国开发 署提供的 7,500万欧元生物多样性保护贷款, 标志 着地方政府与国际组织之间在生物多样性领域的
合作进入了崭新模式; 2016年与北京环境交易所 《生物多样性友好型森林碳汇标准》, 探索出一条 生物多样性融资的创新之路; 2017年，《自然资本议 定书》替代 《生态系统服务与生物多样性经济学》 (TEEB)成为国际发展主流，FECO也正在积极翻译 和出版该议定书。同年初, 生态环境部发布《生物 遗传资源获取与惠益分享管理条例(草案)》, 作为中 国履行《名古屋议定书》的重要举措。

尽管已取得上述重要进展, 但总体而言, 中国 仍然对《公约》提供的各类资源(如企业贡献《爱知 目标》框架导则和实践案例), 正在进行的各种项目 (如自然资本核算、商品生产影响指标和信息披露), 正在变化的机制(推动国家加入GPBB)、体制(秘书 处新增企业事务官员职位与预算), 以及正在演变 的企业有关谈判与决议等仍缺乏深度研究和理解。

2020年将在北京召开《公约》缔约方大会第15 次会议(COP-15), 这对促进企业参与GPBB的机制 化构建和服务平台搭建提出了更高要求。企业的参 与将有力地推动中国在国际政治经济格局中从旁 观者、参与者到引领者的角色转换, 同时有利于增 加转移支付资金、推动科技创新、产业升级与绿色 供应链等生态文明建设任务。中国应积极研究和利 用GPBB平台上的各种资源与机会, 研究、吸收和借 鉴国际经验，推动CBBP机制建设，加快推进国内 企业参与生物多样性、私营部门主流化等工作。

\section{对中国的政策建议}

2015年中国加入GPBB国际机制, 对推动国内 企业参与生物多样性保护产生了积极促进作用。但 与进展较快的国家相比, 中国并没有参与到国际重 大方法学、工具与标准的开发过程中, 中国国内相 关领域成果和企业实践案例也没有被介绍和纳入 到GPBB平台的网络中。如何充分利用该资源网络, 以及如何引导中国企业、行业协会、学术研究机构 和非政府组织更好地参与、贡献并受益于 《公约》 资源体系, 仍有大量工作要做。

结合中国国情和在生物多样性保护方面的重 点工作, 提出如下建议:

(1)在制度贡献方面，应加强政策研究，研提 COP-15企业参与新决议草案。中国应充分利用公约 资源网络平台, 深入研究公约大会历经数届通过的 企业相关决议，包括全球伙伴关系、国家与区域倡 
议、企业贡献爱知目标、生物多样性和生态系统服 务价值评估、信息披露等, 确保新的企业决议有的 放矢、循序渐进并且符合国际发展趋势。总结企业 贡献《爱知目标》的成果和不足, 利用 2020 年 COP-15的时间节点, 承前启后地提出《公约》新十 年企业战略，尤其注意深化与《联合国可持续发展 目标》(SDG)的内在关联性，提出全球企业通过参与 《公约》而贡献SDG的技术路线图与时间表。

(2)在机制建设方面, 积极参与和逐渐引领 GPBB机制建设，协力支撑“一带一路”等中国倡议。 深刻理解《公约》秘书处顶层设计的深远用意, 并 预判发展动向。支持秘书处继续推动更多国家、地 区加入GPBB机制。紧密配合秘书处促进成员国之 间沟通协作。积极参与GPBB主席国和执行委员会 竞选, 参加、新建和领导有利于中国的工作组, 支 持每年 “企业全球论坛” 等活动。加强和其他成员国 交流与合作，探索利用GPBB机制和平台的20多个 国家与区域成员优势，支持“一带一路”、“南南合 作”和“企业走出去”等议程。

(3)在人员保障方面, 加强人才队伍建设, 提升 引领能力。针对 《公约》秘书处已增设企业事务官 员的新变化, 建议中国国内增编相关工作人员, 具 体负责与秘书处和全球伙伴关系各成员国之间的 沟通与合作, 加强中国在该机制中的作用, 促进中 国国内企业、行业学习、运用公约资源网络, 推动 中国各部门的最佳实践被纳入到该网络中传播和 借鉴。有条件的情况下, 还可考虑为秘书处输送技 术支持人员。

(4)在GPBB平台方面, 加强研究借鉴和技术参 与, 为 $\mathrm{GPBB}$ 平台建设提供技术支持。借鉴国际 GPBB平台网络提供的有关信息, 支持加快研究中 国和全球企业参与生物多样性保护的方法学与工 具开发。具体措施包括: (a)加强已有GPBB平台网络 提供的相关研究成果的翻译和引进, 促进相关部 门、行业通过制定指引、指南或在已有规划中更多 纳入生物多样性保护目标; (b)鼓励国内企业进行先 进试点示范, 利用GPBB平台推广宣传最佳实践案 例; (c)通过鼓励国内公司试点, 推荐中方专家加入
和支持相关研讨会在华召开等方式，支持中国参与 GPBB平台重大方法学(工具)开发并做出贡献; (d) 探索继承发扬《爱知目标》、符合《联合国可持续 发展议程》且协同增效的“2020后企业参与十年”国 际纲领性文件或战略框架。

\section{参考文献}

Wang AH, Wu JY, Liu JX (2015) Business and biodiversity: A review of the negotiation progress of a new issue under the Convention on Biological Diversity. Biodiversity Science, 23, 689-694. (in Chinese with English abstract) [王爱华, 武建勇, 刘纪新 (2015) 企业与生物多样性: 《生物多样 性公约》新议题的产生与谈判进展. 生物多样性, 23, 689-694.]

Zhang FC, Fang J, Yin GF (2014) Enterprises' participation in biodiversity: Question, situation and approach. China WTO Tribune, (11), 71-75. (in Chinese) [张风春，方菁，殷格非 (2014) 企业参与生物多样性的问题、现状与路径. WTO 经济导刊, (11), 71-75.]

Zhang FC, Liu WH (2015) Multi-stakeholder engaging mechanism for biodiversity conservation in China. Environmental Protection, (5), 29-33. (in Chinese) [张风春, 刘文慧 (2015) 生物多样性保护多方利益相关者参与现 状与机制构建研究. 环境保护, (5), 29-33.]

Zhao Y, Sun Z, Wang Y (2018a) Rio Tinto Group: The creativity in Biodiversity Bank. WTO Tribune, (3), 45-46. (in Chinese) [赵阳, 孙祯, 王影 (2018) 力拓集团: “生物 多样性银行”的创意. WTO经济导刊, (3), 45-46.]

Zhao Y, Wang Y (2018) Why should companies consider natural capital? WTO Tribune, (9), 34-36. (in Chinese) [赵 阳, 王影 (2018) 企业为什么需要考量自然资本? WTO 经济导刊, (9), 34-36.]

Zhao Y, Wen YY, Yang LR, Li HT (2018b) Learning from international experience to promote the Global Partnership Between Business and Biodiversity (GPBB) in China. Biodiversity Science, 26, 1249-1254. (in Chinese with English abstract) [赵阳，温源远，杨礼荣，李宏涛 (2018) 推动中国企业参与《生物多样性公约》全球伙伴关系的 机制建设. 生物多样性, 26, 1249-1254.]

Zhuang GT, Shen HB (2013) Biodiversity conservation: New problems and challenges. World Environment, (4), 16-21. (in Chinese) [庄国泰, 沈海滨 (2013) 生物多样性保护面 临的新问题和新挑战. 世界环境, (4), 16-21.]

(责任编委: 薛达元 责任编辑: 时意专) 